\title{
Migrating Azygos Vein and Vanishing Azygos Lobe: MDCT Findings
}

\section{Alberto Villanueva ${ }^{1}$ \\ Jose Cáceres ${ }^{2}$ \\ Mónica Ferreira ${ }^{1}$ \\ Jordi Broncano ${ }^{1}$ \\ Esther Pallisa ${ }^{2}$ \\ Gorka Bastarrika ${ }^{1}$}

Keywords: azygos lobe, azygos vein, MDCT

DOI:10.2214/AJR.09.3303

Received July 9, 2009; accepted after revision September 12, 2009.

'Department of Radiology, Clínica Universidad de Navarra, 36 Avenida Pío XII, 31008 Pamplona, Spain. Address correspondence to $A$. Villanueva (avillanueva@unav.es).

${ }^{2}$ Department of Radiology, Hospital Vall d’Hebron, Barcelona, Spain.

\section{AJR2010; 194:599-603}

0361-803X/10/1943-599

(c) American Roentgen Ray Society

OBJECTIVE. The purpose of this study was to describe six cases of migrating azygos vein and to explain the etiologic factors that contribute to the migration. Six patients with migrating azygos vein were studied by MDCT before and after migration. Five patients had right pneumothorax. All patients had repeated episodes of cough, vomiting, and a short mesoazygos.

CONCLUSION. Pneumothorax, increased intrathoracic pressure, and a short mesoazygos, in combination or alone, are the main factors in azygos vein migration.

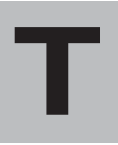

he azygos lobe is a common variant that is usually well seen on a chest radiograph as a pleural line (azygos fissure) that crosses the apex of the right lung. The azygos vein is visible in the lowermost part of the fissure as a tear-shaped shadow. On CT studies, the fissure is clearly visible, and the azygos vein is seen as a thicker structure following the same path as the fissure.

Occasionally, the azygos vein may escape from the fissure and migrate to its usual position in the mediastinum, adjacent to the right wall of the trachea, leaving the azygos fissure empty. Rarely, the azygos fissure may disappear as well (i.e., vanishing azygos lobe)

The migrating azygos vein has been described in isolated case reports. To our knowledge, there are no reported cases of vanishing azygos lobe. In this article, we describe six new cases of migrating azygos vein, one of them with a vanishing azygos lobe, and attempt to explain the etiologic factors that contribute to migration of the vein. We believe that the cause is multifactorial, with most patients, but not all, undergoing previous episodes of pneumothorax of the right lung. Persistent cough and vomiting and a short fissure may also be contributing factors, because not all the patients had pneumothorax.

\section{Materials and Methods}

Six patients, five men and one woman (age range, $34-80$ years; mean age, 58.8 years), with migrating azygos vein were studied. The condition was detected prospectively in four patients over an 8-month period, from March to November
2008, within our daily clinical practice. Two cases were diagnosed retrospectively: one was found while searching specifically for migrating azygos vein in a group of 130 patients who underwent surgery of the right lung, and the other was found incidentally while reviewing a series of patients with radiation pneumonitis.

For each patient, the clinical history, MDCT images, and available chest radiographs were studied to investigate evidence of pneumothorax of the right lung and other conditions that can produce increased intrathoracic pressure (e.g., vomiting or cough) or upward traction of the azygos vein (e.g., apical pulmonary fibrosis).

All patients were examined using 64-MDCT. The studies were performed in deep inspiration and breath-holding after administration of 100-120 mL of IV contrast medium. In all cases, the images underwent reconstructions at $1.3,3$, or $5 \mathrm{~mm}$ thickness in axial, sagittal, and coronal planes using mediastinal, bone, and lung windows. The position of the trigone (i.e., the triangular area delimiting the upper part of the azygos fissure) and fissure and the position of the azygos vein before and after migration were studied.

For all patients, CT studies performed before the diagnosis were available; these included single-slice nonhelical CT for two patients, singledetector helical CT for two others, and MDCT for the last two patients. Posteroanterior and lateral radiographs before migration were available for four patients, and radiographs taken after the diagnosis were available for five patients.

In each case, the shape of the azygos lobe was classified according to Boyden's protocol [1] into types $\mathrm{A}, \mathrm{B}$, and $\mathrm{C}$, on the basis of the position of the trigone. In type $\mathrm{A}$, the trigone is situated in the 

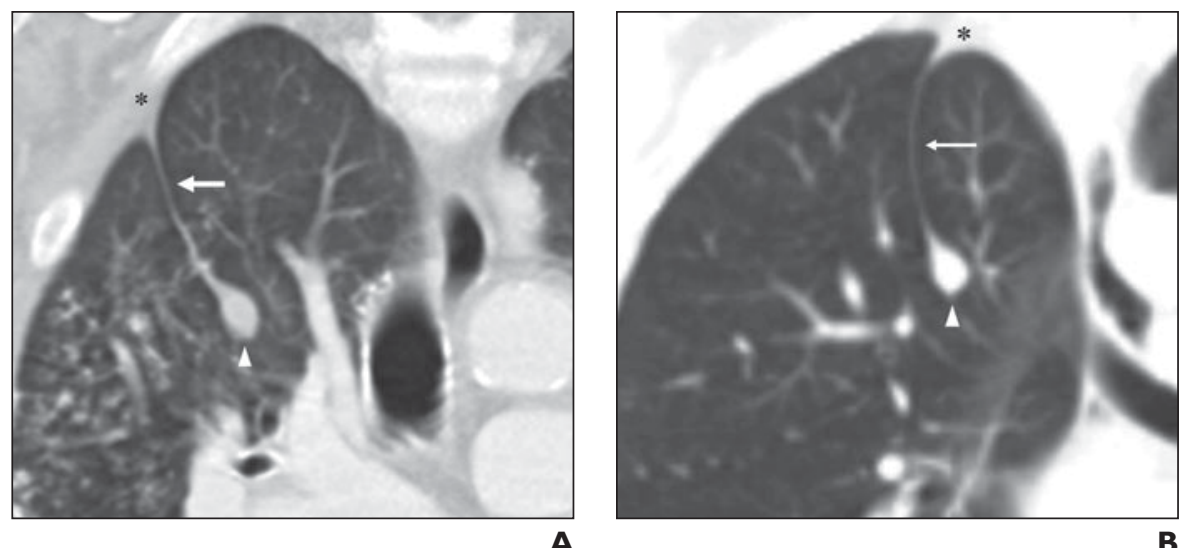

A

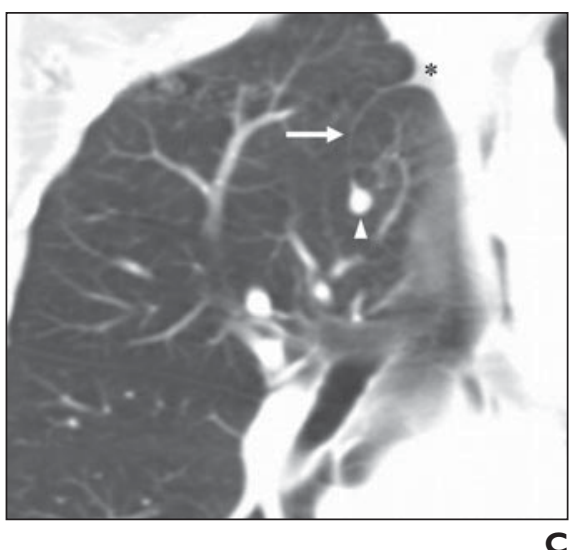

C

Fig. 1-Three types of azygos lobe, as seen on conventional chest radiography.

A-C, Images show azygos fissure (arrow), azygos vein (arrowhead), and trigone (asterisk) in azygos lobe type A (A), type B (B), and type C (C).

lateral aspect of the pulmonary apex; in type B, the trigone is located at the midpoint of the apex, and the fissure is more or less vertical; and in type $\mathrm{C}$, the trigone is located medially, and the fissure curves toward the mediastinum. The length of the fissure varies with the type of lobe; it is longer in type A and shorter in types B and C (Fig. 1).

Images were reviewed by two chest radiologists, one with 32 years of experience and the other with 12 years of experience. There was agreement between them in all cases.

\section{Results}

MDCT scans showed an absence of the azygos vein within the fissure in all patients. Prior CT evidence of its presence inside the fissure was available in all cases. In five patients, the vein had migrated to the mediastinum, and in the remaining patient it occupied an intermediate position in the upper chest

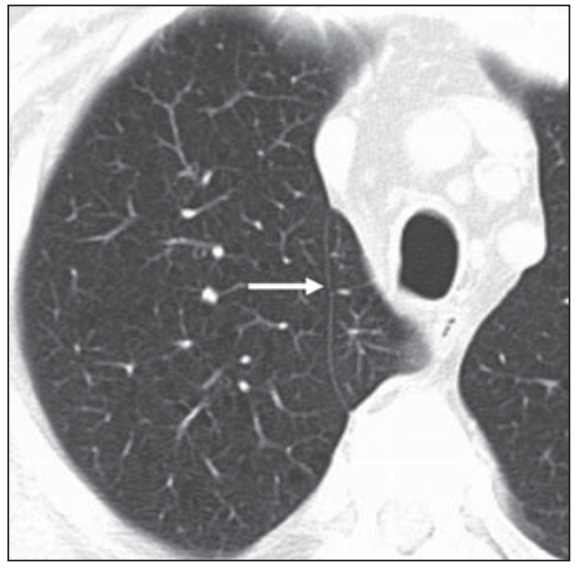

A wall, halfway between its original location and the mediastinum. In one patient, disappearance of the fissure was also shown.

A right-sided pneumothorax was documented in five patients; documentation was made by radiography alone for two patients, by $\mathrm{CT}$ and radiography for two others, and by the clinical records for one patient. The pneumothorax had resulted from fine-needle puncture, lobectomy, or video-assisted thoracoscopic resection of pulmonary nodules. In one patient, two prior pneumothoraces had occurred before azygos vein migration. MDCT studies had been performed during each episode of pneumothorax because of the interventional procedures. MDCT 3 months after the last pneumothorax showed no pneumothorax and the vein inside the fissure.

For one patient, pneumothorax was not documented. The patient had undergone

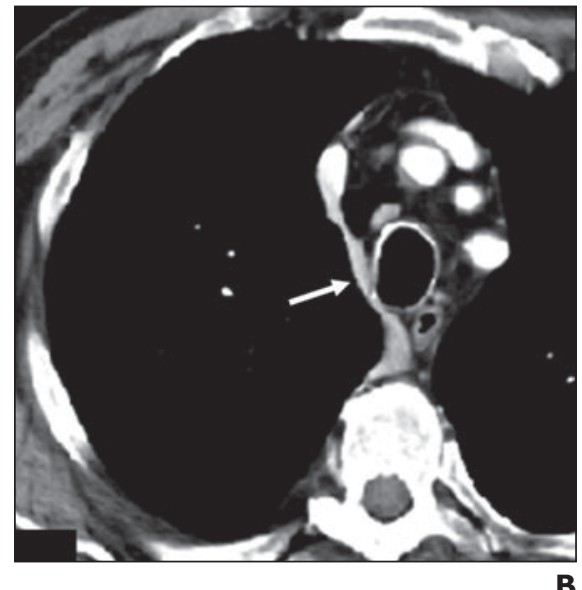

gastrectomy 5 months before the migrating azygos vein was identified. All patients, including the one with no evidence of pneumothorax, had repeated episodes of intense cough or persistent vomiting.

The time interval from the first CT study and CT evidence of azygos vein migration ranged from 3 to 50 months (mean, 14.8 months).

The shape of the azygos lobe before azygos vein migration to the mediastinum was classified as type $\mathrm{C}$ in three cases, type $\mathrm{B}$ in two cases, and type A in one case. After migration, there were no changes in the shape of the fissure in five patients. In the remaining patient, who had a type $\mathrm{C}$ lobe, the fissure disappeared.

\section{Discussion}

The azygos lobe is a congenital variant with an incidence of about $0.4-1.2 \%$ of the population [2]. It occurs when the azygos vein pen-

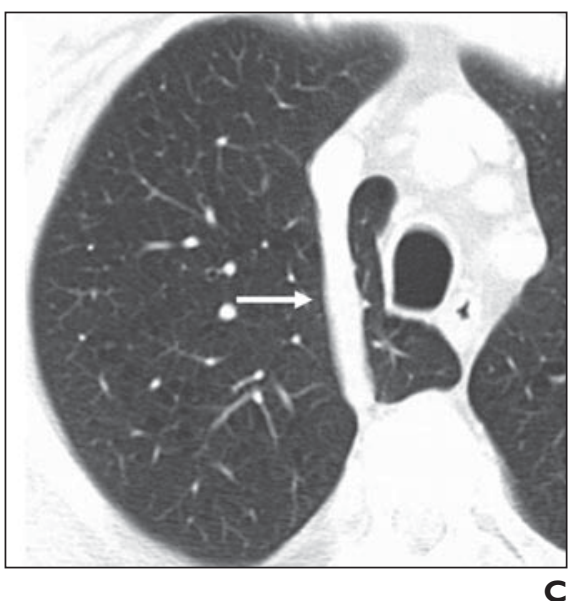

Fig. 2-66-year-old man with cholangiocarcinoma. Intense vomiting was documented in July and August 2008. Patient had pneumothorax 2 years earlier secondary to lung biopsy for pulmonary abscess.

A, Axial MDCT image (August 2008) shows empty azygos fissure (arrow).

B, Image (August 2008) shows azygos vein (arrow) in paramediastinal location.

C, Three months before $\mathbf{A}$ and $\mathbf{B}$ were obtained, azygos vein (arrow) was inside fissure. 
etrates the lung, carrying along pleural layers (mesoazygos) that entrap a portion of the right upper lobe. The resulting azygos lobe is usually well seen on chest radiographs, where it is limited by a pleural line (azygos fissure) that crosses the apex of the right lung. The azygos vein is visible in the lowermost part of the fissure as a tear-shaped shadow. On MDCT, the azygos vein is seen as a thicker structure following the same path as the fissure [3, 4]. The position of the azygos arch is higher than when it follows an intramediastinal course.

The visceral and parietal layers of pleura forming the mesoazygos are not fused, as is shown by the common occurrence of pleural effusion extending into the azygos fissure. The same is true in the case of a pneumothorax: air separates the two pleural layers, leaving the azygos "floating" in the space created. This situation favors mobility of the azygos vein and enables it to jump from its usual position in the fissure and migrate to the mediastinum, with the fissure remaining in place (Figs. 2A and 2B). Because the repositioned azygos vein is joined to a mesentery-like structure, its new location is higher than the normal anatomic path of the intramediastinal azygos vein (Fig. 2C).
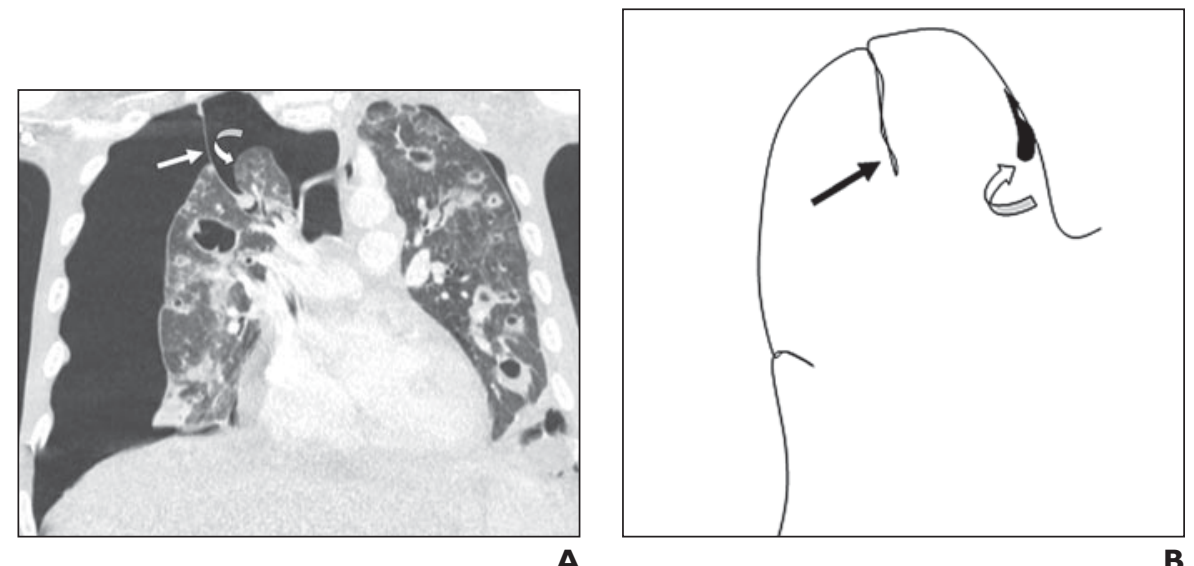

Maldjian and Phatak [5] have offered an explanation for this occurrence based on comments by Milne [6] in relation to cases of azygos lobe associated with a previous pneumothorax. According to Maldjian and Phatak, when a pneumothorax occurs, the lung partially collapses, accompanied by the layer of visceral pleura. In these circumstances, the azygos vein, which is covered by the parietal layer of the fissure, can be released and migrate toward the mediastinum. If the double visceral layer of the pleura maintains its structure without separating when the lung expands, the fissure remains, and the lung maintains its former

A

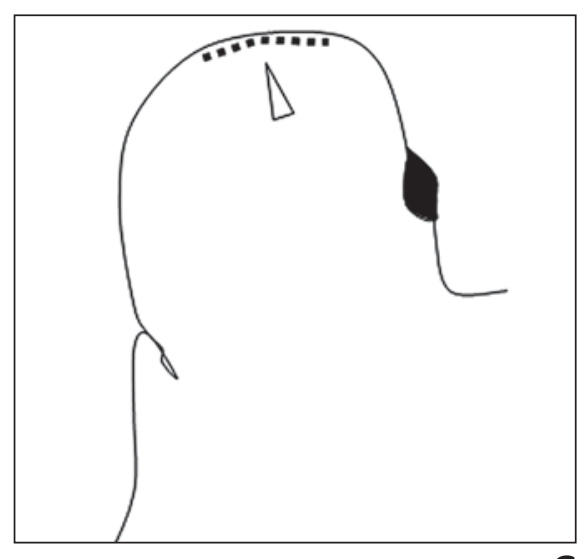

Fig. 3-36-year-old man with right pneumothorax secondary to septic emboli.

A, Coronal reconstruction shows separation of layers of visceral pleura (curved arrow) with parietal pleura and azygos (straight arrow) floating between layers.

B, According to Maldjian and Phatak [5], this separation of layers of visceral pleura facilitates migration of vein to mediastinum (curved arrow), leaving empty fissure (straight arrow) when lung reexpands.

C, If visceral pleura stretches toward lung apex, empty fissure (arrowhead) disappears. In this patient, there was no migration of azygos vein after reexpansion of lung. Dotted line shows theoretical position of stretched fissure.

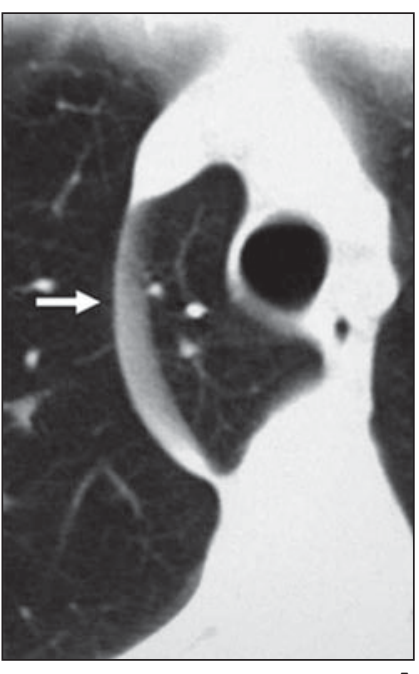

A

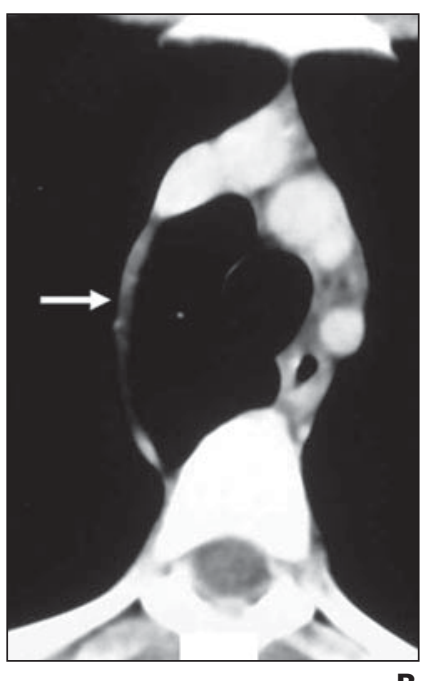

B
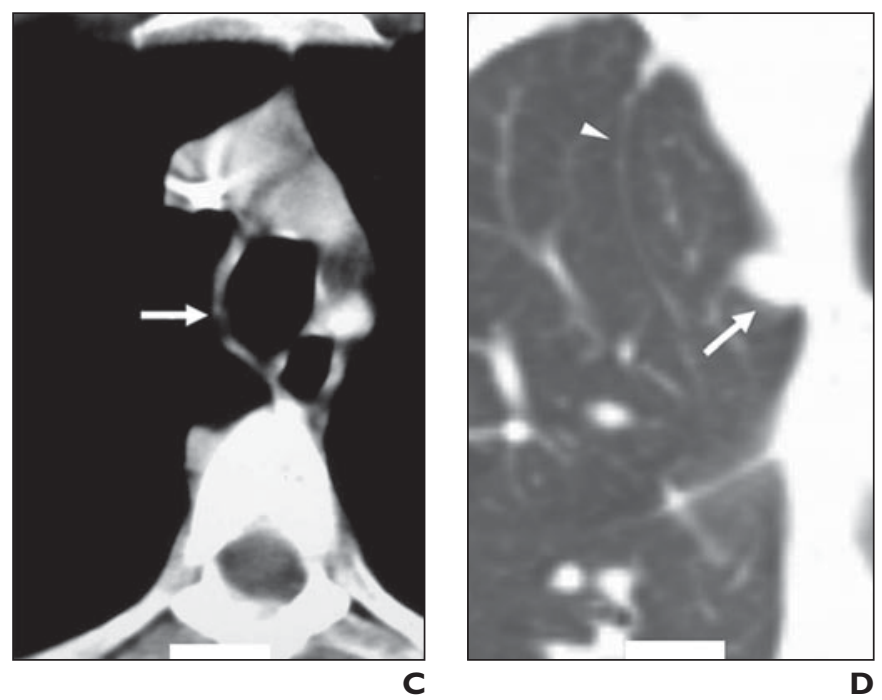

D

Fig. 4-34-year-old woman with resected gastric carcinoma. No pneumothorax was documented

A and B, Postsurgical CT images obtained in April and July 2000 show azygos vein (arrows) in fissure. Patient had intense vomiting between May and September, coinciding with chemotherapy.

C, CT scan obtained in October 2000 shows that vein (arrow) has migrated to mediastinum.

D, MDCT coronal reconstruction in October 2008 shows empty azygos fissure (arrowhead) and vein (arrow) in mediastinum in higher position than usual. 


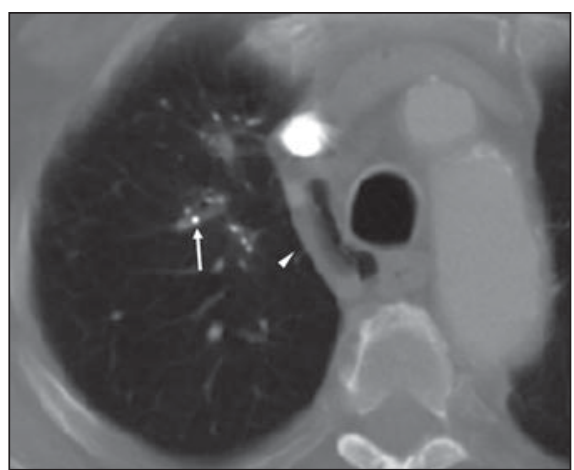

A

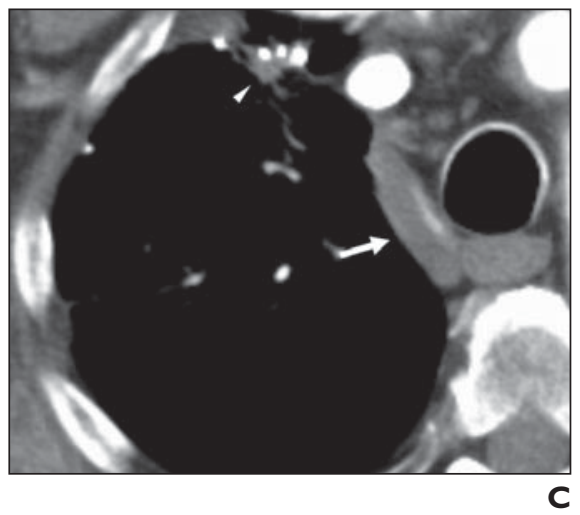

shape, preserving the azygos lobe but without the vein at the inferior aspect. In contrast, if the visceral pleura fold detaches and separates, it will stretch and cover the lung apex, thereby obliterating the fissure. This second mechanism is less common than the former (Fig. 3).

This theory may be correct, but it does not explain azygos migration toward the mediastinum when there is no pneumothorax. Drakonaki et al. [7] reported a migrating azygos vein without evidence of pneumothorax, which occurred after partial collapse of the fourth thoracic vertebra. The authors hypothesized that the kyphosis produced with T4 collapse would verticalize the fissure and favor migration of the vein.

Migration without previous pneumothorax occurred in one of our patients (Fig. 4). The patient had experienced persistent vomiting before migration of the vein. It can be hypothesized that the increase in intrathoracic pressure during recurrent vomiting could make the vein pop out like a watermelon seed pressed by the fingers. We believe that increased intrathoracic pressure plays an important role in the cause of this condition. All of our patients had a history of persistent cough or continuous vomiting. All of them had cancer and were receiving chemotherapy or radiotherapy, which often cause cough and vomiting.
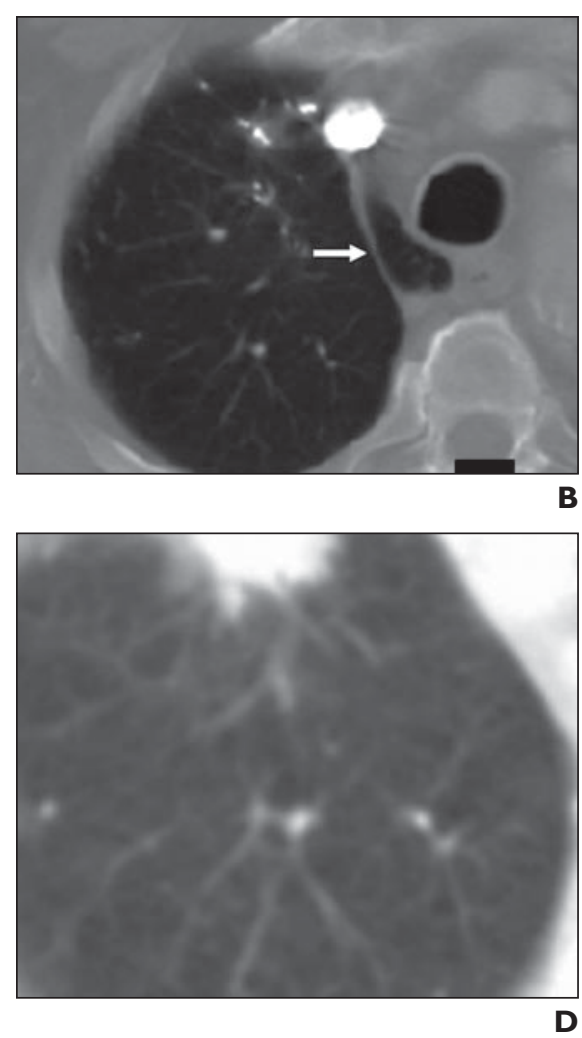

B

Another finding from our series that has not been reported previously is retention of the azygos vein within the fissure after pneumothorax. One of our patients experienced two episodes of pneumothorax within a period of 2 months. The azygos vein did not migrate after the first episode and remained within the fissure up to at least 3 months after the second (Fig. 5B), which supports our theory that the presence of a pneumothorax alone is not necessarily the cause of azygos vein migration [5-9].

It is plausible that the length of the azygos lobe would also have an influence on azygos vein migration. Smaller lobes with shorter fissures conceivably would facilitate migration of the vein, because the path is shorter. Verticalization of the fissure, as suggested by Drakonaki et al. [7] may also play a role in the migration. All of our patients had a short mesoazygos, including the only patient with a type A lobe (Fig. 6). This is a subjective impression, since, to our knowledge, there are no published measurements of the length of the mesoazygos.

We believe that the presence of a pneumothorax is an important etiologic factor in azygos vein migration but not the only one. It is likely that the cause is multifactorial. The pneumothorax would contribute by separation of the two layers of the fissure, but other factors, such as sudden increases in the intratho-
Fig. 5-Vanishing azygos lobe in 80-year-old man with small lung neoplasm, treated with percutaneous implantation of palladium seeds. Patient had right pneumothorax after needle biopsy and second pneumothorax during placement of seeds.

A, CT scan obtained 3 months after procedure shows that several seeds have migrated to arteries of upper lobe (arrow). Azygos vein remains in fissure (arrowhead) after both pneumothoraces occurred. B, Fissure (arrow) is well seen.

C, Six months after $\mathbf{A}$ and $\mathbf{B}$ were obtained, azygos vein (arrow) has migrated to mediastinum. Vascular seeds (arrowhead) have moved forward and upward as consequence of radiation fibrosis.

D, Fissure is not seen. We believe that apical fibrosis pulled fissure upward, blending it with visceral pleura. .

racic pressure (e.g., caused by cough or vomiting) or verticalization of the fissure associated with a vertebral deformity, could also favor migration of the vein. The presence of pneumothorax, increased intrathoracic pressure, and a short mesoazygos, in combination or alone, would be the main factors resulting in azygos vein migration. Thus, the existence of a right-sided pneumothorax in a patient with an azygos lobe does not necessarily lead to azygos vein migration, as was evident in one of our patients.

Disappearance of the azygos lobe (vanishing azygos lobe) occurred in one patient with a type $\mathrm{C}$ lobe. The patient had undergone interstitial brachytherapy for a tumor of the right upper lung, with implantation of palladium-103 seeds that migrated and occluded the arteries of this lung segment. In this case, we believe that the pneumothorax produced during radioactive seed implantation facilitated migration of the azygos vein. Apical pulmonary fibrosis secondary to migration of the implants may have acted as an adjuvant cause, retracting the fissure upward and resulting in its disappearance. To date, no imaging documentation of vanishing azygos lobe has been published. The case presented is the first shown by MDCT images.

We believe that MDCT plays an important role in the discovery of azygos vein migration. 
MDCT of Azygos Vein and Lobe

Fig. 6-54-year-old man with melanoma. A, Coronal MDCT reconstruction shows type A lobe with short fissure (arrow). Vein is in fissure (arrowhead). One month later, patient underwent resection of metastasis in right lung and experienced intense vomiting after surgery.

B, Three months after A was obtained, coronal reconstruction shows empty fissure (straight arrow) and vein in apex of lung (curved arrow).

C and D, Axial slices confirm empty fissure and atypical location of vein (arrows).
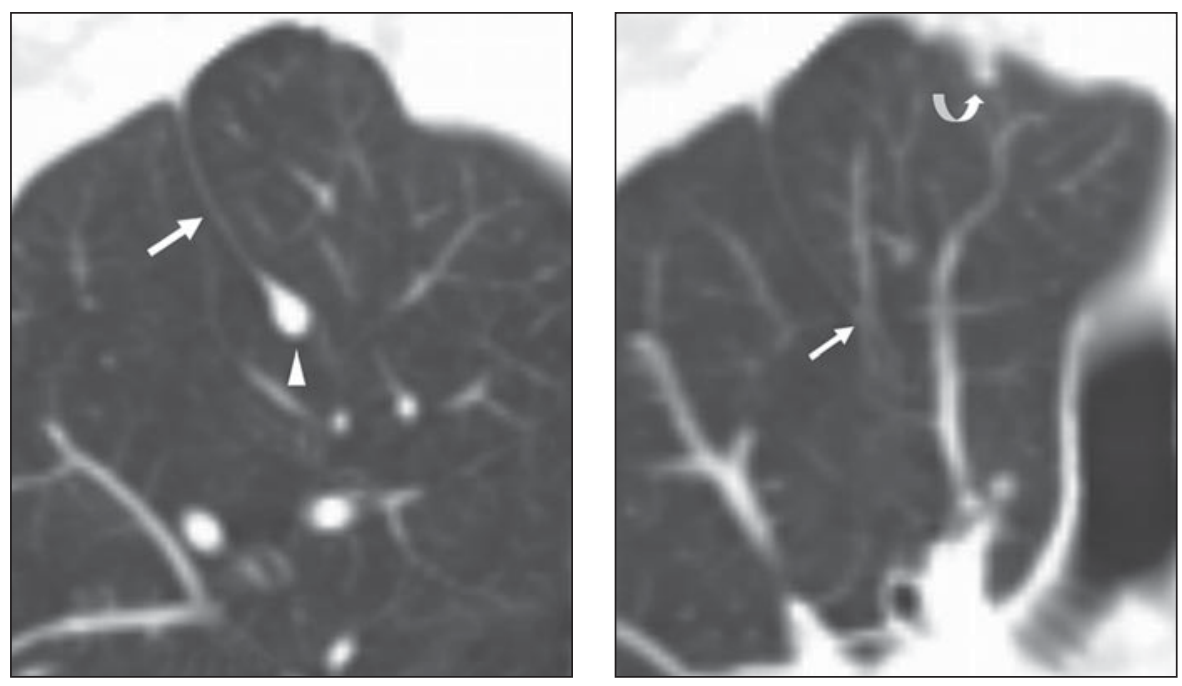

A B

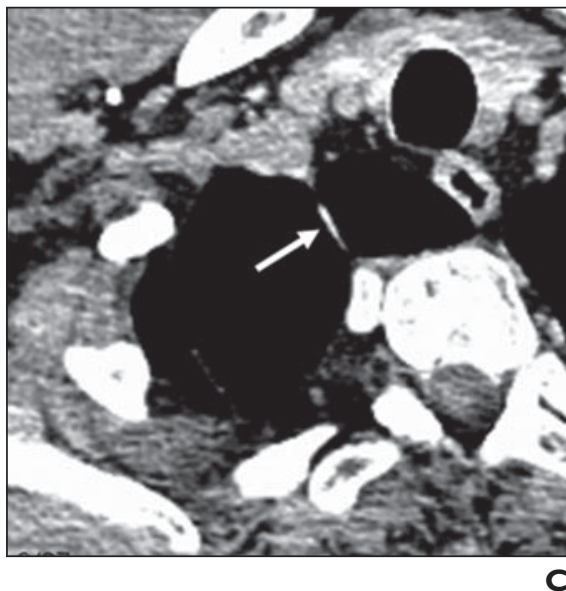

To summarize, our experience indicates

The fact that all our patients were examined with MDCT enabled easy visualization of the azygos vein and the fissure in three spatial planes. This may be why four of our six cases of migration were found within a period of 8 months and suggests that this entity may occur more often than is currently believed.

Moreover, MDCT reconstructions enabled documentation of the gradual migration of the azygos vein. In one patient, the vein was visualized halfway toward the mediastinum in the lung apex, a finding that has not been shown previously, to our knowledge (Fig. 6).

On the basis of the MDCT findings reported here, azygos vein migration can be classified into three groups: first, cases in which the empty fissure persists and the vein is relocated to a mediastinal position; second, cases in which the fissure persists, migration is partial, and the azygos is located in a position halfway to the mediastinum; and third, cases in which the azygos vein migrates and the mesoazygos disappears. that azygos vein migration from the fissure to the mediastinum may not be an uncommon occurrence. MDCT is an optimal method for diagnosing this condition because of its capability to perform millimetric reconstructions in all three planes. Although pneumothorax is common in the history of patients with migrating azygos vein, we believe that this entity is a progressive event with a multifactorial cause. Azygos vein migration with disappearance of the fissure is rare. Identification of this phenomenon may have some forensic value.

\section{References}

1. Boyden EA. The distribution of bronchi in gross anomalies of the right upper lobe, particularly lobes subdivided by the azygos vein and those containing pre-eparterial bronchi. Radiology 1952; 58:797-807

2. Arakawa T, Terashima T, Miki A. A human case of an azygos lobe: determining an anatomical basis for its therapeutic postural drainage. Clin Anat

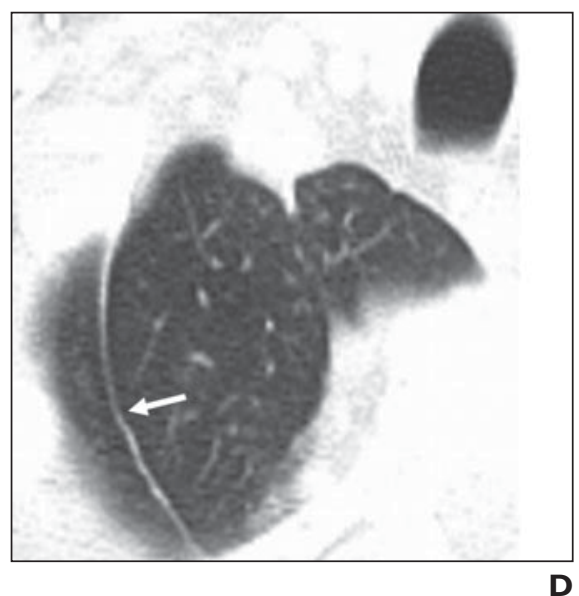

2008; 21:524-530

3. Mata J, Cáceres J, Alegret X, Coscojuela P, De Marcos JA. Imaging of the azygos lobe: normal anatomy and variations. AJR 1991; 156:931-937

4. Speckman JM, Gamsu G, Webb WR. Alterations in CT mediastinal anatomy produced by an azygos lobe. AJR 1981; 137:47-50

5. Maldjian PD, Phatak T. The empty azygos fissure: sign of an escaped azygos vein. $J$ Thorac Imaging 2008; 23:54-56

6. Milne ENC. Editor's response. J Thorac Imaging 1986; 4:vii-ix

7. Drakonaki EE, Voloudaki A, Daskalogiannaki M, Karantanas AH, Gourtsoyiannis N. Migratory azygos vein: a case report. J Comput Assist Tomogr 2008; 32:99-100

8. Asai K, Urabe N, Takeichi H. Spontaneous pneumothorax and a coexistent azygos lobe. Jpn J Thorac Cardiovasc Surg 2005; 53:604-606

9. Betschart T, Goerres GW. Azygos lobe without azygos vein as a sign of previous iatrogenic pneumothorax: two case reports. Surg Radiol Anat 2009; 31:559-562 